\section{Dr. Balevic, et al reply}

\section{To the Editor:}

We read with great interest the very thoughtful commentary by Joob and Wiwanitkit ${ }^{1}$. We agree with the authors that, despite conflicting clinical data to date, it is possible that hydroxychloroquine (HCQ) may have a protective effect in the setting of the coronavirus disease 2019 (COVID-19). More importantly, we agree that optimal HCQ dosage and timing is a critical underpinning for clinical trials. However, we highlight several considerations regarding treatment with high-dose HCQ.

To optimize dosing, the relationship between drug concentration in the target tissue and response must be well characterized. Because the precise mechanism of action of HCQ for SARS-CoV-2 is unknown, current dosing strategies are based on extrapolating the in vitro antiviral activity targets (e.g., effective concentration (EC) 50, EC100]. Herein lies one of the first challenges: the reported EC50 target for inhibiting viral replication varies in different reports $(0.72-17.31 \mu \mathrm{M})^{2,3}$. Based on in vitro EC50 targets, dosages of HCQ $400 \mathrm{mg}$ orally every $12 \mathrm{~h}$ for 2 doses followed by $200 \mathrm{mg}$ every $12 \mathrm{~h}$ to achieve target unbound lung concentrations has been proposed $^{2}$. However, the authors relied on animal-derived lung partition coefficients for HCQ, and it was unclear how they accounted for differences in the fraction of unbound drug in tissue compared to plasma ${ }^{2}$. Differences in these assumptions significantly alter whether the proposed dosing would achieve target concentrations ${ }^{4}$. Similarly, we modeled total serum concentrations and observed that only the lowest EC50 target $(0.72 \mu \mathrm{M})$ is achievable in serum using proposed loading doses, and all concentrations were significantly less than those needed for complete viral inhibition ${ }^{5}$.

Although our analysis suggests that most in vitro target concentrations cannot be achieved in plasma and pulmonary interstitial fluid even with higher dosing, this does not preclude a potential benefit for HCQ in the setting of COVID-19. In addition to possible antiinflammatory benefits, intracellular lung concentrations may be critical for the drug's potential antiviral effect. HCQ appears to block intracellular transport of SARS-CoV-2, with only $0.03 \%$ of virions in HCQ-treated cells localizing to endolysosomes, compared to $34.3 \%$ in untreated cells $s^{3}$. Because animal studies suggest substantially higher HCQ concentrations in lung tissue compared to plasma, it is possible that antiviral target concentrations can be achieved in the lung cells compared to interstitial fluid with conventional dosing ${ }^{3,6}$. However, because HCQ accumulates in lung tissue over several months ${ }^{6}$, the duration of therapy (as opposed to the dose) may be the key to understanding the drug's potential in COVID-19. In addition, we are aware of early reports that suggest patients with systemic lupus erythematosus and SARS-CoV-2 infection develop severe COVID-19 at a similar frequency regardless of prior HCQ use, underscoring the importance of further study?

Stephen J. Balevic ${ }^{1} \mathbb{D}$, MD, MHS

Christoph P. Hornik ${ }^{2}$ (D), MD, PhD

Thomas P. Green ${ }^{3}$, MD, MS

Megan E.B. Clowse ${ }^{1}$ (I) $, \mathrm{MD}, \mathrm{MPH}$

Daniel Gonzalez ${ }^{4}$, PharmD, PhD

Anil R. Maharaj², PhD

Laura E. Schanberg ${ }^{2}$, MD

Amanda M. Eudy ${ }^{1}$ D, PhD

Geeta K. Swamy ${ }^{5}$, MD

Brenna L. Hughes ${ }^{5}$, MD, MSc

Michael Cohen-Wolkowiez ${ }^{2}$ D $, \mathrm{MD}, \mathrm{PhD}$

${ }^{1}$ Department of Rheumatology and Immunology, Duke University School

of Medicine, Durham, North Carolina;

${ }^{2}$ Duke Clinical Research Institute, Durham, North Carolina;

${ }^{3}$ Department of Pediatrics, Northwestern University, Feinberg School of
Medicine, Evanston, Illinois;

${ }^{4}$ Division of Pharmacotherapy and Experimental Therapeutics, UNC Eshelman School of Pharmacy, The University of North Carolina at Chapel Hill, Chapel Hill, North Carolina;

${ }^{5}$ Division of Maternal-Fetal Medicine, Department of Obstetrics \& Gynecology, Duke University School of Medicine, Durham, North Carolina, USA.

Salary and/or research support for this project was provided by the Rheumatology Research Foundation's Scientist Development Award, the Thrasher Research Fund, the Childhood Arthritis and Rheumatology Research Alliance/Arthritis Foundation, the Derfner Foundation, NIGMS/NICHD (2T32GM086330-06), NICHD (5R01-HD07667604, HHSN275201000003I), and a Duke Health/ Private Diagnostic Clinic ENABLE grant.

Dr. Schanberg serves on the data and safety monitoring board for Sanofi (sarilumab). Sanofi is a maker of hydroxychloroquine. Dr. Swamy chairs the Independent Data Monitoring Committee for GlaxoSmithKline (RSV vaccine trials). Dr. Cohen-Wolkowiez receives support for research from industry for drug development in adults and children.

Address correspondence to Dr. S.J. Balevic, Department of Rheumatology and Immunology, Duke University School of Medicine, 2301 Erwin Road, CHC, T-Level, Durham, NC 27710, USA. Email: stephen.balevic@duke.edu.

\section{REFERENCES}

1. Joob B, Wiwanitkit V. Evidence of protective effect of hydroxychloroquine on COVID-19. J Rheumatol 2020;47:1587.

2. Yao X, Ye F, Zhang M, Cui C, Huang B, Niu P, et al. In vitro antiviral activity and projection of optimized dosing design of hydroxychloroquine for the treatment of severe acute respiratory syndrome coronavirus 2 (SARS-CoV-2). Clin Infect Dis $2020 \mathrm{Mar} 9$ (E-pub ahead of print).

3. Liu J, Cao R, Xu M, Wang X, Zhang H, Hu H, et al. Hydroxychloroquine, a less toxic derivative of chloroquine, is effective in inhibiting SARS-CoV-2 infection in vitro. Cell Discov 2020;6:16.

4. Arnold SL, Buckner F. Hydroxychloroquine for treatment of SARS-CoV-2 infection? Improving our confidence in a model-based approach to dose selection. Clin Transl Sci 2020 Apr 8 (E-pub ahead of print).

5. Balevic SJ, Hornik CP, Green TP, Clowse ME, Gonzalez D, Maharaj $\mathrm{AR}$, et al. Hydroxychloroquine in patients with rheumatic disease complicated by COVID-19: clarifying target exposures and the need for clinical trials. J Rheumatol 2020 May 11 (in press).

6. McChesney EW. Animal toxicity and pharmacokinetics of hydroxychloroquine sulfate. Am J Med 1983;75:11-18.

7. Konig MF, Kim AH, Scheetz MH, Graef ER, Liew JW, Simard J, et al; COVID-19 Global Rheumatology Alliance. Baseline use of hydroxychloroquine in systemic lupus erythematosus does not preclude SARS-CoV-2 infection and severe COVID-19. Ann Rheum Dis 2020 May 7 (E-pub ahead of print). 